\title{
Probes for 4th Generation Constituents of Dark Atoms in Higgs Boson Studies at the LHC
}

\author{
M. Yu. Khlopov ${ }^{1,2,3}$ and R. M. Shibaev ${ }^{1}$ \\ ${ }^{1}$ National Research Nuclear University "Moscow Engineering Physics Institute", Moscow 115409, Russia \\ ${ }^{2}$ Centre for Cosmoparticle Physics "Cosmion" Moscow 115409, Russia \\ ${ }^{3}$ APC laboratory 10, rue Alice Domon et Léonie Duquet, 75205 Paris Cedex 13, France
}

Correspondence should be addressed to R. M. Shibaev; ratibor.shibaev@list.ru

Received 20 October 2013; Revised 18 December 2013; Accepted 18 December 2013; Published 18 February 2014

Academic Editor: Konstantin Belotsky

Copyright (C) 2014 M. Yu. Khlopov and R. M. Shibaev. This is an open access article distributed under the Creative Commons Attribution License, which permits unrestricted use, distribution, and reproduction in any medium, provided the original work is properly cited. The publication of this article was funded by SCOAP ${ }^{3}$.

\begin{abstract}
The nonbaryonic dark matter of the Universe can consist of new stable charged species, bound in heavy neutral "atoms" by ordinary Coulomb interaction. Stable $\bar{U}$ (anti-U)quarks of 4th generation, bound in stable colorless $(\bar{U} \bar{U} \bar{U})$ clusters, are captured by the primordial helium, produced in Big Bang Nucleosynthesis, thus forming neutral "atoms" of O-helium (OHe), a specific nuclear interacting dark matter that can provide solution for the puzzles of direct dark matter searches. However, the existence of the 4th generation quarks and leptons should influence the production and decay rates of Higgs boson and is ruled out by the experimental results of the Higgs boson searches at the LHC, if the Higgs boson coupling to 4th generation fermions is not suppressed. Here, we argue that the difference between the three known quark-lepton families and the 4th family can naturally lead to suppression of this coupling, relating the accelerator test for such a composite dark matter scenario to the detailed study of the production and modes of decay of the $125.5 \mathrm{GeV}$ boson, discovered at the LHC.
\end{abstract}

\section{Introduction}

The cosmological dark matter can consist of dark atoms, in which new stable charged particles are bound by ordinary Coulomb interaction (see [1-5] for review and references). In order to avoid anomalous isotopes overproduction, stable particles with charge -1 (and corresponding antiparticles as tera-particles [6]) should be absent [7], so that stable negatively charged particles should have charge -2 only.

There exist several types of particle models, in which heavy stable -2 charged species, $\mathrm{O}^{--}$, are predicted as follows:

(a) AC-leptons, predicted in the extension of standard model, based on the approach of almost-commutative geometry [8-11],

(b) technileptons and antitechnibaryons in the framework of walking technicolor models (WTC) [12-18],

(c) finally, stable "heavy quark clusters" $\bar{U} \bar{U} \bar{U}$ formed by anti- $U$ quark of 4 th generation $[8,19-23]$.
All these models also predict corresponding +2 charge particles. If these positively charged particles remain free in the early Universe, they can recombine with ordinary electrons in anomalous helium, which is strongly constrained in the terrestrial matter. Therefore, cosmological scenario should provide a mechanism, which suppresses anomalous helium. There are two possibilities, requiring two different mechanisms of such suppression.

(i) The abundance of anomalous helium in the Galaxy may be significant, but in the terrestrial matter there exists a recombination mechanism suppressing this abundance below experimental upper limits $[8,10]$. The existence of a new strict $U(1)$ gauge symmetry, causing new Coulomb-like long range interaction between charged dark matter particles, is crucial for this mechanism. Therefore, the existence of dark radiation in the form of hidden photons is inevitable in this approach. 
(ii) Free positively charged particles are already suppressed in the early Universe and the abundance of anomalous helium in the Galaxy is negligible [3, 4, 20].

These two possibilities correspond to two different cosmological scenarios of dark atoms. The first one is realized in the scenario with AC leptons, forming neutral AC atoms [10]. The second assumes charge asymmetric case with the excess of $\mathrm{O}^{--}$, which form atom-like states with primordial helium $[3,4,20]$.

If new stable species belong to nontrivial representations of electroweak SU(2) group, sphaleron transitions at high temperatures can provide the relationship between baryon asymmetry and excess of -2 charge stable species, as it was demonstrated in the case of WTC $[12,24-26]$.

After it is formed in the Standard Big Bang Nucleosynthesis $(\mathrm{SBBN}),{ }^{4} \mathrm{He}$ screens the $\mathrm{O}^{--}$charged particles in composite $\left({ }^{4} \mathrm{He}^{++} \mathrm{O}^{--}\right)$O-helium “atoms" [20].

In all the models of O-helium, $\mathrm{O}^{--}$behaves either as lepton or as specific "heavy quark cluster" with strongly suppressed hadronic interaction. Therefore, O-helium interaction with matter is determined by nuclear interaction of He. These neutral primordial nuclear interacting objects can explain the modern dark matter density and represent a nontrivial form of strongly interacting dark matter [27-35].

The cosmological scenario of O-helium Universe allows to explain many results of experimental searches for dark matter [3]. Such scenario is insensitive to the properties of $\mathrm{O}^{--}$, since the main features of $\mathrm{OHe}$ dark atoms are determined by their nuclear interacting helium shell. It challenges experimental probes for the new stable charged particles at accelerators and such probes strongly depend on the nature of $\mathrm{O}^{--}$.

Stable -2 charge states $\left(\mathrm{O}^{--}\right)$can be elementary like ACleptons or technileptons or look like elementary as technibaryons. The latter, composed of techniquarks, reveal their structure at much higher energy scale and should be produced at the LHC as elementary species. They can also be composite-like "heavy quark clusters" $\bar{U} \bar{U} \bar{U}$ formed by anti- $U$ quark in the models of stable fourth generation $[19,20]$.

In the context of composite dark matter scenario, accelerator probe for new stable quark-lepton generation acquires the meaning of critical test for the existence of charged constituents of cosmological dark matter.

The signature for double charged AC leptons and techniparticles is unique and distinctive what has already allowed to obtain the lower bound on their mass of $430 \mathrm{GeV}$ in the ATLAS experiment [36].

Test for composite $\mathrm{O}^{--}$at the LHC can be only indirect through the search for heavy hadrons, composed of single $U$ or $\bar{U}$ and light quarks (similar to $R$-hadrons) [5], or by virtual effects of 4 th generation fermions in the processes with known particles. Here, we study a possibility for experimental probe of the hypothesis of stable 4th generation in the studies of $125.5 \mathrm{GeV}$ Higgs boson, discovered in the ATLAS [37, 38] and the CMS experiments $[39,40]$ at the LHC. The results of these studies [37-40] indicate that the number of the detected events, being the production cross section times the decay rate of Higgs boson to two-photon channel, is consistent with the prediction of the Standard model. On the other hand, as it was first revealed in [41], the existence of 4 th generation leads to enhancement of the main mechanism of Higgs boson production in $p P$ collisions, what puts constraints on the effect of 4th generation particles and practically excludes the possibility of their full strength coupling to $125.5 \mathrm{GeV}$ Higgs boson.

In the model of stable 4 th generation, the difference of these fermions from the quarks and leptons of the three known families is related to some new conserved charge (which can be even a gauge charge) that protects the stability of the lightest quarks and leptons ( $U$-quark and the 4 th neutrino- $N$ ). The experimental lower limits on the new quarks and leptons make these particles be heavier than the three light families, what can be explained by the existence of an additional mechanism of their mass generation, for example, in the framework of multi-Higgs models. It can naturally lead to suppression of the coupling of 4 th generation fermions to the $125.5 \mathrm{GeV}$ Higgs boson, discovered at the LHC. Here, we explore a possibility to make the 4 th generation hypothesis consistent with the experimental data on the two gamma decays of Higgs boson, what opens the door to the indirect probes of the charged constituents of composite dark matter in the detailed studies of production and modes of decay of the $125.5 \mathrm{GeV}$ Higgs boson.

\section{Effects of 4th Generation in Higgs Boson Production and Decay}

2.1. The Stable 4th Generation. The precision data on the parameters of the $W$ and $Z$ bosons of the Standard model do not exclude [42-45] the existence of the 4 th generation of quarks and leptons.

The existence of the 4 th generation can follow from the heterotic string phenomenology and its difference from the three known light generations can be explained by a new conserved charge, possessed only by its quarks and leptons [19-23, 46-48]. Strict conservation of this charge makes the lightest particle of the 4th family (the 4th neutrino) absolutely stable, but it was shown in [46-48] that this neutrino cannot be the dominant form of the dark matter. The same conservation law requires the lightest quark to be long living $[19,20]$. In principle, the lifetime of $U$ can exceed the age of the Universe, if $m_{U}<m_{D}[19,20]$.

Due to their Coulomb-like QCD attraction $\left(\propto \alpha_{c}^{2}\right.$. $m_{U}$, where $\alpha_{c}$ is the QCD constant) stable double and triple $U$ bound states $(U U q),(U U U)$ can exist $[6,7,19-$ 23]. The corresponding antiparticles can be formed by heavy antiquark $\bar{U}$. Formation of these double and triple states at accelerators and in cosmic rays is strongly suppressed by phase space constraints, but they can be formed in early Universe and strongly influence cosmological evolution of 4 th generation hadrons. As shown in [20], anti- $U$-triple state called anutium or $\Delta_{3 \bar{U}}^{--}$is of a special interest. This stable anti$\Delta$-isobar, composed of $\bar{U}$ antiquarks, can be bound with ${ }^{4} \mathrm{He}$ in atom-like state of O-helium [8]. 


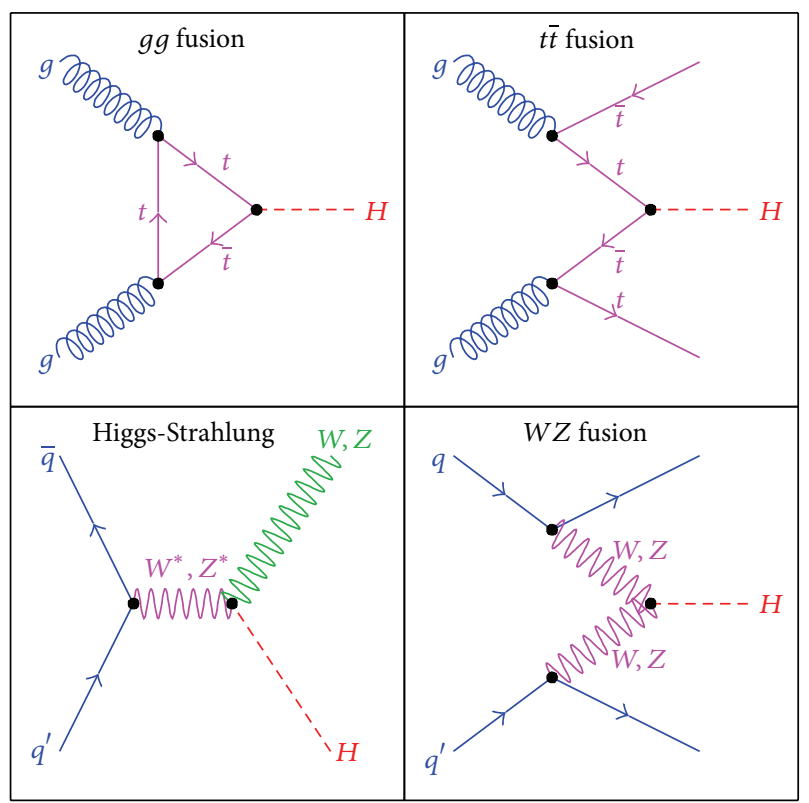

FIGURE 1: Feynman diagrams of the main processes of Higgs boson production in $p p$ collisions (taken from [52]).

Since simultaneous production of three $U \bar{U}$ pairs and their conversion in two doubly charged quark clusters $U U U$ are suppressed, the only possibility to test the models of composite dark matter from 4th generation in the collider experiments is a search for production of stable hadrons containing single $U$ or $\bar{U}$ [5] or for effects of 4 th generation quarks and leptons in the processes with known particles. Such effects should influence the production and decay rates of the Higgs boson, so the first step in such analysis is to check the consistency of the 4th generation hypothesis with the experimental data on the $125.5 \mathrm{GeV}$ Higgs boson, discovered at the LHC. In the present paper, we show that the suppression of couplings of this boson to the 4th generation quarks and leptons can make compatible the experimental data on the two-photon decay mode of the Higgs boson with this hypothesis. The precise determination of the range of such couplings implies the account for the two-loop electroweak corrections and for the results of the Higgs searches in other decay channels, what goes beyond the scope of the present paper.

We take for definiteness the masses of the fourth generation $U$ and $D$ quarks to be about $350 \mathrm{GeV}$, of the lepton $E$ about $100 \mathrm{GeV}$, and the mass of the 4th neutrino $N$ about $50 \mathrm{GeV}$ (see [4] for the recent review and references) and vary the coupling constants of the fourth generation fermions to the $125.5 \mathrm{GeV}$ Higgs boson taking into account their possible suppression.

2.2. Effects of 4th Generation in Higgs Boson Production. The main channels of Higgs boson production in $p p$ collisions are presented in Figure 1. Their relative contribution was calculated in [49] and presented in Figures 2 and 3.

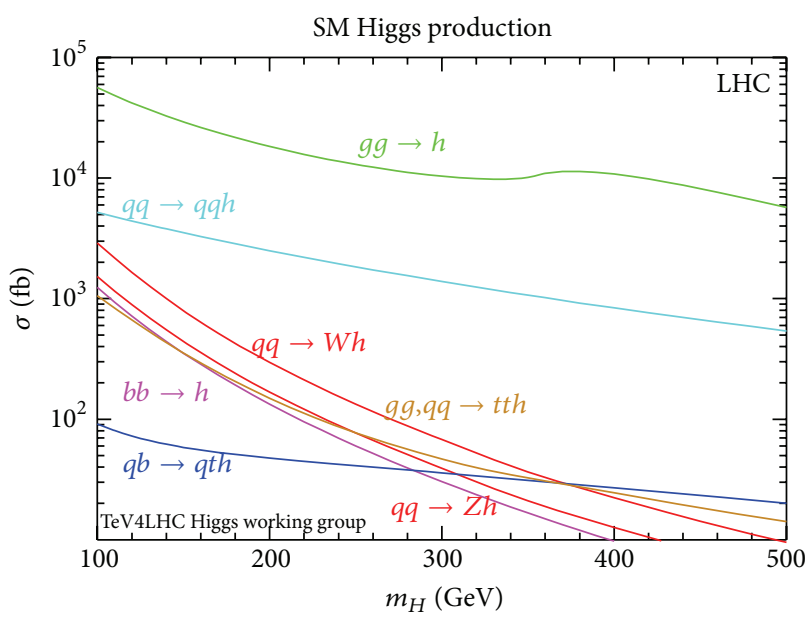

FIgURE 2: The cross section of the main processes of Higgs boson production in $p p$ collisions at LHC (from [49]).

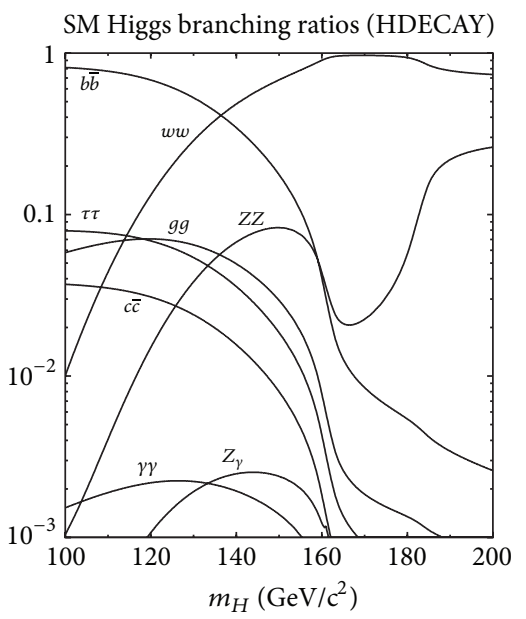

FIGURE 3: The branching ratio of Higgs boson decay modes in the Standard model (from [49]).

The dominant mechanism of Higgs production, related to the gluon-gluon fusion, goes through the loop with virtual quarks. Thus, the existence of the quarks of fourth generation should influence the amplitude of this process. The corresponding contribution depends on the mass of these quarks and on their Yukawa coupling to the Higgs boson. The value of this coupling constant can vary due to the fact that the large mass of the fourth generation fermions can result from a combined effect of several Higgs fields so that the coupling to the $125.5 \mathrm{GeV}$ Higgs boson can be suppressed. In the present work, we calculate the cross section of the Higgs boson production with the use of the HIGLU program [50] for different parameters of the model of 4th generation with the account for the QCD corrections.

2.3. Effects of 4th Generation in Two-Photon Higgs Boson Decay. Effects of 4th generation in the decay rates of Higgs boson, calculated in [41], are presented in Figure 4. The twophoton decay, considered here, goes through a loop diagram, 


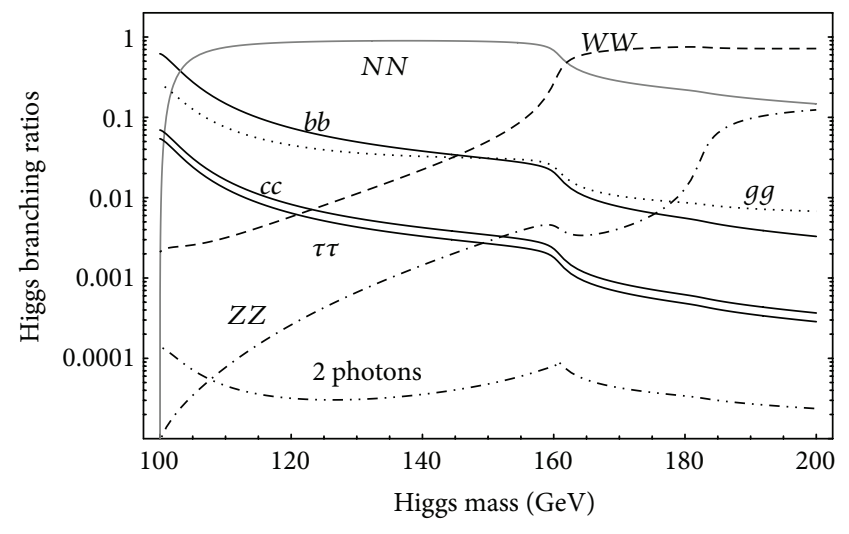

FIGURE 4: Branching ratios of the main processes of Higgs boson decay in the model of stable 4th generation (from [41]).

which contribute all the charged particles. The width of this decay, calculated as in the Standard model [51] and modified with the account for the suppression of Higgs coupling constants, is given by

$$
\Gamma(H \longrightarrow \gamma \gamma)=\frac{G_{F} \alpha^{2} m_{H}^{3}}{128 \sqrt{2} \pi^{3}}\left|\sum_{i=W, f} g_{i} N_{c} e_{i}^{2} F_{i}\right|^{2},
$$

where $\alpha_{s} \approx 0.118$ and $\alpha \approx 1 / 137$ are the QCD and QED running gauge constants and $N_{c}$ and $e_{i}$ are the number of color degrees of freedom and the electric charge of an $i$ th particle, respectively. The loop factor $F_{i}$ is given by

$$
F_{\text {boson }}=2+3 \delta+3 \delta(2-\delta) f(\delta)
$$

for bosons and

$$
F_{\text {fermion }}=-2 \delta(1+(1-\delta) f(\delta))
$$

for fermions, where

$$
f(\delta)= \begin{cases}\operatorname{arctg} \frac{1}{\sqrt{\delta-1}}, & \delta \geq 1 \\ \frac{i}{2} \ln \frac{1+\sqrt{1-\delta}}{1-\sqrt{1-\delta}}+\frac{\pi}{2}, & \delta<1 .\end{cases}
$$

The parameter $\delta$ is defined as

$$
\delta=\left(\frac{2 m_{i}}{m_{H}}\right)^{2} .
$$

In the limit of $m_{i} \gg m_{H}$, the loop factor depends very weakly on the mass of an intermediate particle, so that the width equation (1) depends weakly on the mass of the heavy 4 th generation fermions. In (1), $g_{i}$ are the suppression factors of Higgs boson couplings. They are equal to 1 for the fermions of the three known generations and can vary from 0 to 1 for the quarks and charged lepton of the 4 th generation.

\section{The Results of Calculations}

In the present work, we have calculated the ratio

$$
R=\frac{\sigma_{\mathrm{SM} 4}(g g \rightarrow H) \Gamma_{\mathrm{SM} 4}(H \rightarrow \gamma \gamma)}{\sigma_{\mathrm{SM}}(g g \rightarrow H) \Gamma_{\mathrm{SM}}(H \rightarrow \gamma \gamma)}
$$

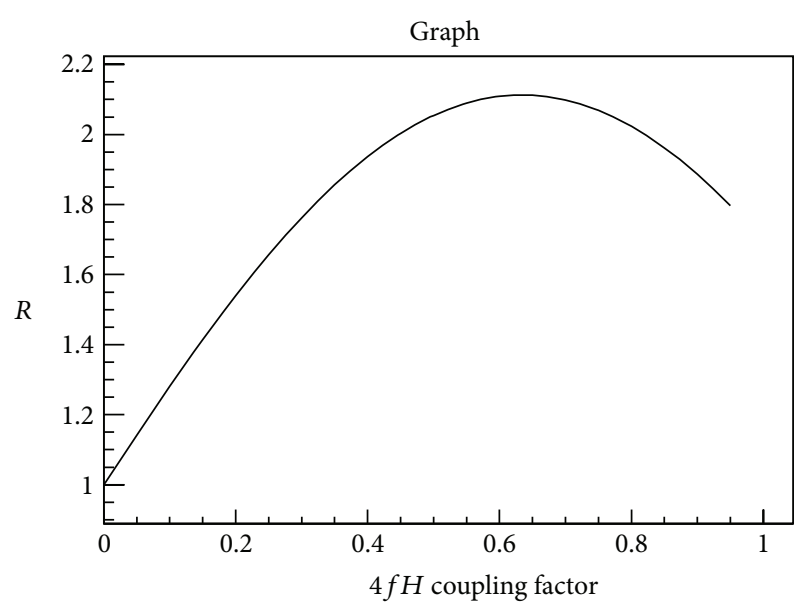

FIgURE 5: The dependence of the ratio $R$ on the suppression factor, which is the same for all the quarks and leptons of the 4 th generation.

of the number of events of two-photon decays of Higgs boson in the model of 4th generation and in the Standard model and compared it with the results of ATLAS $[37,38]$ and CMS experiments $[39,40]$ at the LHC. These results within the experimental errors are consistent with the prediction of the Standard model $(R=1)$ with the median values $R=1.1$ in CMS and $R=1.3$ in ATLAS that may favor some modest excess of the number of events relative to this prediction. One cannot make compatible the prediction of the model of the 4th generation with these results without suppression of Higgs boson coupling to new quarks and leptons.

The following possibilities for the suppression of the Higgs boson couplings were considered.

(a) The suppression factor is the same for all the quarks and leptons of the 4 th generation.

(b) The uptype and downtype fermions of the 4th generation have different suppression factors.

(c) Quarks and leptons of the 4th generation have different suppression factors.

(d) All the fermions of the 4th generation have different suppression factors.

The results of calculations are presented in Figures 5, 6, $7,8,9$, and 10 . The predicted number of events is strongly enhanced by the effects of 4th generation quarks in gluon fusion mechanism of Higgs boson production, so that the corresponding suppression of the Higgs couplings is necessary to make the number of events close to the prediction of the Standard model. However, in the case of different suppression factors for different types of 4th generation particles, a nontrivial range of these parameters is possible. One can expect that the analysis of the predictions of the model of the 4th generation for the number of events of other decay modes of the $125.5 \mathrm{GeV}$ Higgs boson will provide an overdetermined system of equations for these parameters, making possible the complete test of this model. 


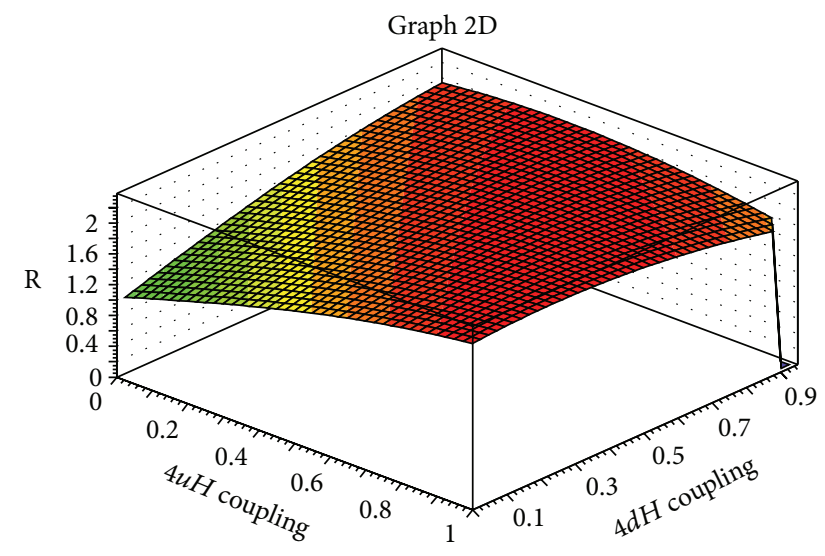

FIgURE 6: The dependence of the ratio $R$ on the suppression factors $4 u H$ and $4 d H$ of Higgs coupling to correspondingly up- and downtype fermions of the 4 th generation.

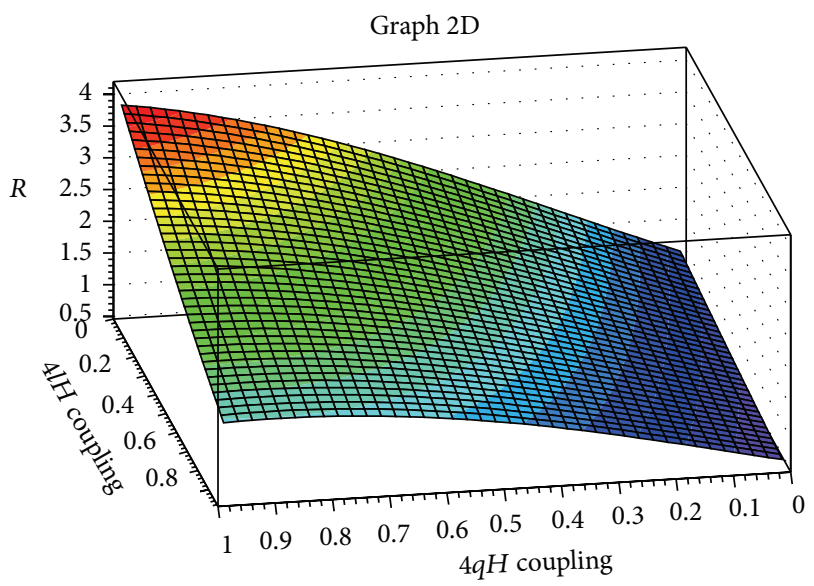

Figure 7: The dependence of the ratio $R$ on the suppression factors $4 q H$ and $4 l H$ of Higgs coupling to correspondingly quarks and leptons of the 4 th generation.

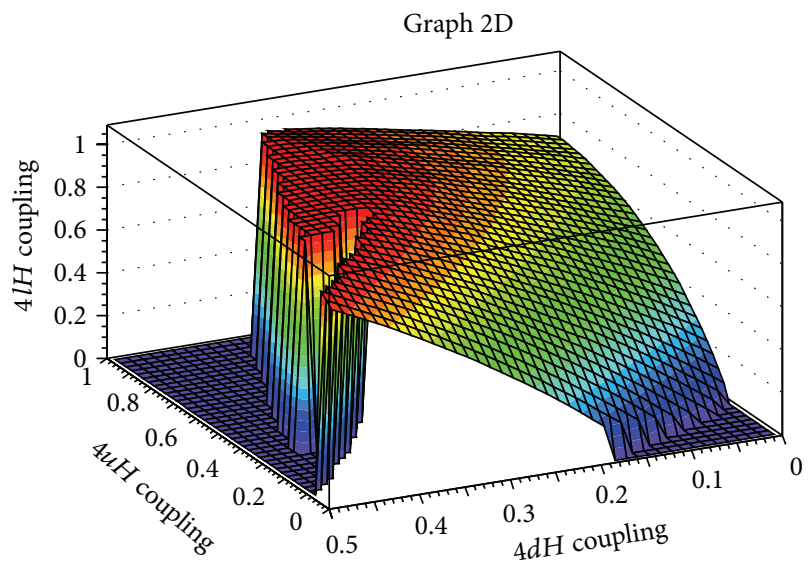

FIGURE 8: The surface in the space of parameters of the suppression factors $4 d H, 4 u H$, and $4 l H$ in the Higgs coupling to the quarks and leptons of the 4th generation, at which the median value of ratio $R=$ 1.3, measured in the ATLAS experiment at LHC [37], is reproduced.

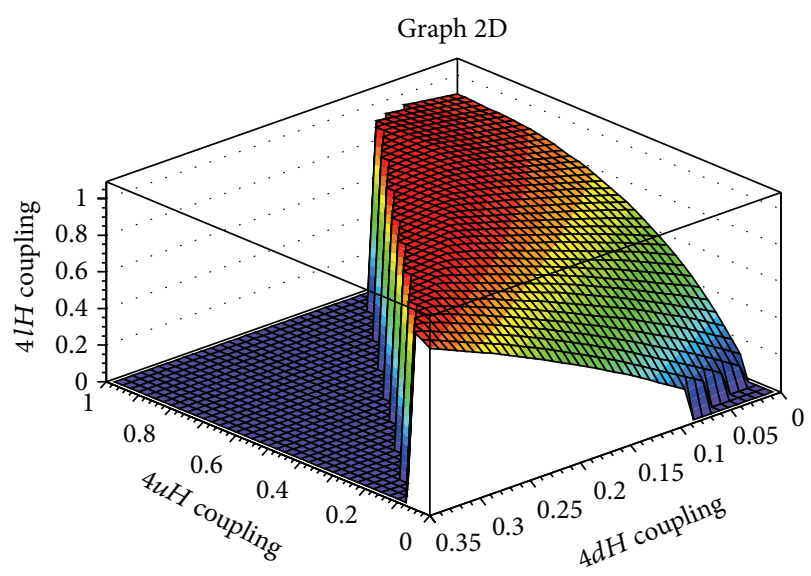

FIGURE 9: The surface in the space of parameters of the suppression factors $4 d H, 4 u H$, and $4 l \mathrm{H}$ in the Higgs coupling to the quarks and leptons of the 4 th generation, at which the median value of ratio $R=$ 1.1, measured in the CMS experiment at LHC [40], is reproduced.

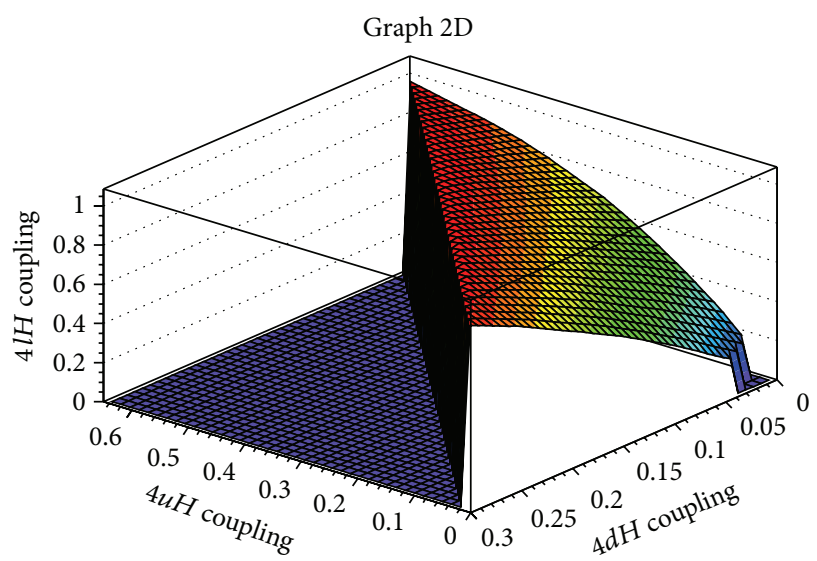

FIGURE 10: The surface in the space of parameters of the suppression factors $4 d H, 4 u H$, and $4 l H$ in the Higgs coupling to the quarks and leptons of the 4th generation, at which the value of ratio $R=1$, corresponding to the Standard model prediction, is reproduced.

\section{Conclusions}

The cosmological dark matter can be formed by stable heavy double charged particles bound in neutral OHe dark atoms with primordial He nuclei by ordinary Coulomb interaction. This scenario sheds new light on the nature of dark matter and offers nontrivial solution for the puzzles of direct dark matter searches. It can be realized in the model of stable 4th generation and challenges for its experimental probe at accelerators. In the context of this scenario, search for effects of new heavy quarks and leptons acquires the meaning of direct experimental probe for charged constituents of dark atoms of dark matter.

The $\mathrm{O}^{--}$constituents of $\mathrm{OHe}$ in the model of stable 4th generation are "heavy antiquark clusters" $\bar{U} \bar{U} \bar{U}$. Production of such clusters (and their antiparticles) at accelerators is virtually impossible. Therefore experimental test of the hypothesis of stable 4 th generation is reduced to the search for 
stable or metastable $U$ hadrons, containing only single heavy quark or antiquark, or to the studies of virtual effects of 4 th generation quarks and leptons in the processes with known particles.

The discovery of the $125.5 \mathrm{GeV}$ Higgs boson at the LHC opens the new room for such indirect test of the model of stable 4th generation. The number of detected events of decays of this boson to the two-photon channel is consistent within the experimental errors with the prediction of the Standard model, putting severe constraints on the contribution of new quarks and leptons. On the other hand, the existence of heavy quarks of the 4th generation should lead to enhancement of the gluon fusion mechanism of Higgs boson production, which is its dominant production mechanism in $p p$ collisions. Therefore, to be compatible with the experimental data, the model of the stable 4 th generation should involve a mechanism of suppression of new quark and lepton couplings to the $125.5 \mathrm{GeV}$ Higgs boson [53]. Taking into account the difference of the 4th generation from the three known families of quarks and leptons and in particular the lower limits on the masses of new quarks and leptons, one can assume some additional mechanisms of their mass generation, what can lead to suppression of their couplings to the $125.5 \mathrm{GeV}$ Higgs boson.

In the present work, we have shown that, indeed, the suppression in the Higgs boson couplings to 4th generation quarks and lepton the can make compatible the existence of this generation with the experimental data on the two-photon decays of the $125.5 \mathrm{GeV}$ Higgs boson. We consider this result as the first step in the thorough investigation of the predictions of the model of stable 4th generation for the whole set of decay channels of Higgs boson. The confrontation of these predictions with the detailed experimental study of the 125.5 GeV Higgs boson will provide the complete test for the composite dark matter scenarios based on the model of the stable 4th generation.

\section{Conflict of Interests}

The authors declare that there is no conflict of interests regarding the publication of this paper.

\section{Acknowledgment}

The authors would like to thank K. Belotsky for discussions.

\section{References}

[1] M. Y. Khlopov, A. G. Mayorov, and E. Y. Soldatov, "Puzzles of dark matter in the light of dark atoms," Journal of Physics, vol. 309, Article ID 012013, 2011.

[2] M. Y. Khlopov, A. G. Mayorov, and E. Y. Soldatov, "Dark Atoms of the Universe: towards OHe nuclear physics," Bled Workshops in Physics, vol. 11, p. 73, 2010.

[3] M. Y. Khlopov, "Physics of dark matter in the light of dark atoms," Modern Physics Letters A, vol. 26, no. 38, pp. 2823-2839, 2011.

[4] M. Yu. Khlopov, "Fundamental particle structure in the cosmological dark matter," International Journal of Modern Physics A, vol. 28, Article ID 1330042, 2013.
[5] K. M. Belostky, M. Y. Khlopov, and K. I. Shibaev, "Probes for constituents of composite dark matter," Gravitation and Cosmology, vol. 18, pp. 127-131, 2012.

[6] S. L. Glashow, "A sinister extension of the standard model to $\mathrm{SU}(3) \mathrm{XSU}(2) \mathrm{XSU}(2) \mathrm{XU}(1)$," http://arxiv.org/abs/hep-ph/ 0504287.

[7] D. Fargion and M. Khlopov, “Tera-leptons' shadows over sinister universe," Gravitation and Cosmology, vol. 19, no. 4, pp. 219-231, 2013.

[8] M. Y. Khlopov, "New symmetries in microphysics, new stable forms of matter around us," http://arxiv.org/abs/astro-ph/ 0607048

[9] C. A. Stephan, "Almost-commutative geometries beyond the standard model," Journal of Physics A, vol. 39, Article ID 9657, 2006.

[10] D. Fargion, C. A. Stephan, D. Fargion et al., "Dark matter with invisible light from heavy double charged leptons of almostcommutative geometry?" Classical and Quantum Gravity, vol. 23, Article ID 7305, 2006.

[11] A. Connes, Noncommutative Geometry, Academic Press, London, UK, 1994.

[12] M. Y. Khlopov and C. Kouvaris, "Strong interactive massive particles from a strong coupled theory," Physical Review D, vol. 77, no. 6, Article ID 065002, 2008.

[13] F. Sannino and K. Tuominen, "Orientifold theory dynamics and symmetry breaking," Physical Review D, vol. 71, Article ID 051901, 2005.

[14] D. K. Hong, S. D. H. Hsu, and F. Sannino, "Composite Higgs from higher representations," Physics Letters B, vol. 597, no. 1-2, pp. 89-93, 2004.

[15] D. D. Dietrich and F. Sannino, "Light composite Higgs boson from higher representations versus electroweak precision measurements: predictions for CERN LHC," Physical Review D, vol. 72, Article ID 055001, 2005.

[16] D. D. Dietrich, F. Sannino, and K. Tuominen, "Light composite Higgs and precision electroweak measurements on the resonance: an update," Physical Review D, vol. 73, Article ID 037701, 2006.

[17] S. B. Gudnason, C. Kouvaris, and F. Sannino, "Towards working technicolor: effective theories and dark matter," Physical Review $D$, vol. 73, Article ID 115003, 2006.

[18] S. B. Gudnason, C. Kouvaris, and F. Sannino, "Dark matter from new technicolor theories," Physical Review D, vol. 74, Article ID 095008, 2006.

[19] K. M. Belotsky, D. Fargion, M. Y. Khlopov, R. V. Konoplich, M. G. Ryskin, and K. I. Shibaev, "Heavy hadrons of 4th family hidden in our Universe and close to detection?" Gravitation and Cosmology, vol. 11, pp. 41-42, 2005.

[20] M. Yu. Khlopov, "Composite dark matter from the fourth generation," Journal of Experimental and Theoretical Physics Letters, vol. 83, no. 1, pp. 1-4, 2006.

[21] K. Belotsky, M. Khlopov, and K. Shibaev, "Stable matter of 4th generation: hidden in the Universe and close to detection?" http://arxiv.org/abs/astro-ph/0602261.

[22] K. M. Belotsky, M. Y. Khlopov, and K. I. Shibaev, "Composite dark matter and its charged constituents," Gravitation and Cosmology, vol. 12, p. 1, 2006.

[23] K. Belotsky, M. Yu. Khlopov, and K. I. Shibaev, "Stable quarks of the 4th family?" in The Physics of Quarks: New Research, N. L. Watson and T. M. Grant, Eds., vol. 265 of Horizons in World Physics, pp. 19-47, NOVA Publishers, Hauppauge, NY, USA, 2009, http://arxiv.org/abs/0806.1067. 
[24] M. Y. Khlopov and C. Kouvaris, "Composite dark matter from a model with composite Higgs boson," Physical Review D, vol. 78, no. 6, Article ID 065040, 2008.

[25] M. Y. Khlopov, “The puzzles of dark matter searches," AIP Conference Proceedings, vol. 1241, p. 388, 2010.

[26] M. Y. Khlopov, A. G. Mayorov, and E. Y. Soldatov, "Composite dark matter and puzzles of dark matter searches," International Journal of Modern Physics D, vol. 19, no. 8-10, pp. 1385-1395, 2010.

[27] B. D. Wandelt, R. Dave, G. R. Farrar et al., "Self-interacting dark matter," http://arxiv.org/abs/astro-ph/0006344.

[28] P. C. McGuire and P. J. Steinhardt, "Cracking open the window for strongly interacting massive particles as the halo dark matter," in Proceedings of the International Cosmic Ray Conference, p. 1566, Hamburg, Germany, 2001, http://arxiv.org/abs/astro$\mathrm{ph} / 0105567$.

[29] G. Zaharijas and G. R. Farrar, "Window in the dark matter exclusion limits," Physical Review D, vol. 72, no. 8, Article ID 083502, 2005.

[30] C. B. Dover, T. K. Gaisser, and G. Steigman, "Cosmological constraints on new stable hadrons," Physical Review Letters, vol. 42, no. 17, pp. 1117-1120, 1979.

[31] S. Wolfram, "Abundances of new stable particles produced in the early universe," Physics Letters B, vol. 82, no. 1, pp. 65-68, 1979.

[32] G. D. Starkman, A. Gould, R. Esmailzadeh, and S. Dimopoulos, "Opening the window on strongly interacting dark matter," Physical Review D, vol. 41, no. 12, pp. 3594-3603, 1990.

[33] D. Javorsek, D. Elmore, E. Fischbach et al., "New experimental limits on strongly interacting massive particles at the TeV scale," Physical Review Letters, vol. 87, Article ID 231804, 2001.

[34] S. Mitra, "Uranus's anomalously low excess heat constrains strongly interacting dark matter," Physical Review D, vol. 70, no. 10, Article ID 103517, pp. 1-103517, 2004.

[35] G. D. Mack, J. F. Beacom, G. Bertone et al., "Towards closing the window on strongly interacting dark matter: far-reaching constraints from Earth's heat flow," Physical Review D, vol. 76, Article ID 043523, 2007.

[36] G. Aad, T. Abajyan, B. Abbott et al., "Search for long-lived, multi-charged particles in $p p$ collisions at $\sqrt{s}=7 \mathrm{TeV}$ using the ATLAS detector," Physics Letters B, vol. 722, no. 4-5, pp. 305323, 2013.

[37] S. M. Consonni, "Higgs search at ATLAS," http://arxiv.org/abs/ 1305.3315 .

[38] G. Aad, T. Abajyan, B. Abbott et al., "Measurements of Higgs boson production and couplings in diboson final states with the ATLAS detector at the LHC," Physics Letters B, vol. 726, no. 1-3, pp. 88-119, 2013.

[39] S. Chatrchyan, V. Khachatryan, A. M. Sirunyan et al., "Observation of a new boson with mass near $125 \mathrm{GeV}$ in pp collisions at $\sqrt{s}=7$ and $8 \mathrm{TeV}$,' Journal of High Energy Physics, vol. 6, p. 81, 2013.

[40] C. Palmer and C. M. S. Collaboration, "CMS measurements of the higgs-like boson in the two photon decay channel," http://arxiv.org/abs/1305.3654.

[41] K. M. Belotsky, D. Fargion, M. Khlopov et al., "Invisible Higgs boson decay into massive neutrinos of fourth generation," Physical Review D, vol. 68, Article ID 054027, 2003.

[42] M. Maltoni, V. A. Novikov, L. B. Okun, A. N. Rozanov, and M. I. Vysotsky, "Extra quark-lepton generations and precision measurements," Physics Letters B, vol. 476, no. 1-2, pp. 107-115, 2000 .
[43] V. A. Ilyin, M. Maltoni, V. A. Novikov, L. B. Okun, A. N. Rozanov, and M. I. Vysotsky, "On the search for $50 \mathrm{GeV}$ neutrinos," Physics Letters B, vol. 503, no. 1-2, pp. 126-132, 2001.

[44] V. A. Novikov, L. B. Okun, A. N. Rozanov, and M. I. Vysotsky, "Extra generations and discrepancies of electroweak precision data," Physics Letters B, vol. 529, no. 1-2, pp. 111-116, 2002.

[45] V. A. Novikov, L. B. Okun, A. N. Rozanov et al., "Mass of the higgs versus fourth generation masses," Journal of Experimental and Theoretical Physics Letters, vol. 76, pp. 127-130, 2002.

[46] K. M. Belotsky, M. Yu. Khlopov, and K. I. Shibaev, “Sakharov's enhancement in the effect of 4th generation neutrino," Gravitation and Cosmology Supplement, vol. 6, pp. 140-143, 2000.

[47] K. M. Belotsky, D. Fargion, M. Y. Khlopov, R. V. Konoplich, and K. I. Shibaev, "Heavy neutrinos of 4 th generation in searches for dark matter," Gravitation and Cosmology, vol. 11, p. 16, 2005.

[48] K. M. Belotsky, D. Fargion, M. Y. Khlopov, and R. V. Konoplich, "May heavy neutrinos solve underground and cosmic-ray puzzles?” Physics of Atomic Nuclei, vol. 71, no. 1, pp. 147-161, 2008.

[49] S. Bolognesi, G. Bozzi, and A. Di Simone, "Higgs at LHC," Nuovo Cimento B, vol. 123, pp. 499-508, 2008.

[50] M. Spira, "HIGLU: a program for the calculation of the total Higgs production cross-section at hadron colliders via gluon fusion including QCDcorrections," http://arxiv.org/abs/hep$\mathrm{ph} / 9510347$.

[51] L. B. Okun, Leptons and Quarks, Nauka, Moscow, Russia, 1990.

[52] http://en.wikipedia.org/wiki/ATLAS_experiment.

[53] O. Eberhardt, G. Herbert, H. Lacker et al., "Impact of a Higgs boson at a mass of $126 \mathrm{GeV}$ on the standard model with three and four fermion generations," Physical Review Letters, vol. 109, no. 24, Article ID 241802, 5 pages, 2012. 

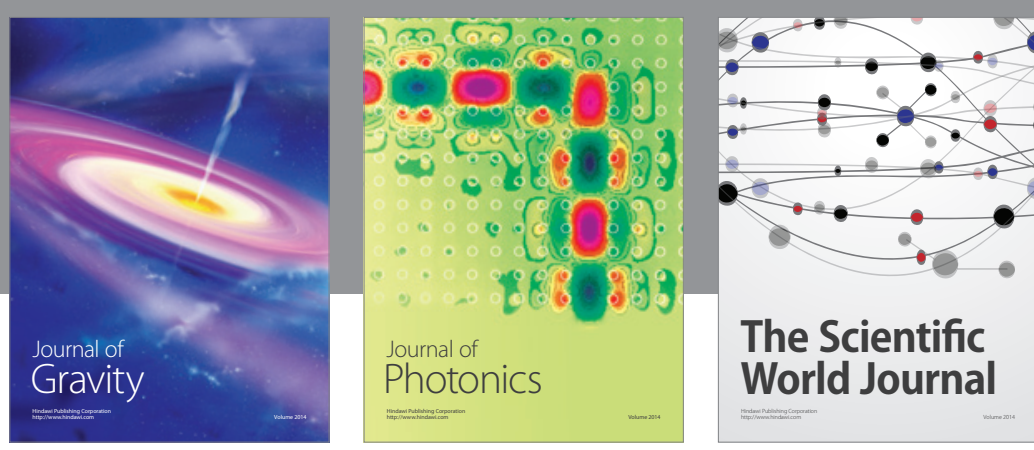

The Scientific World Journal
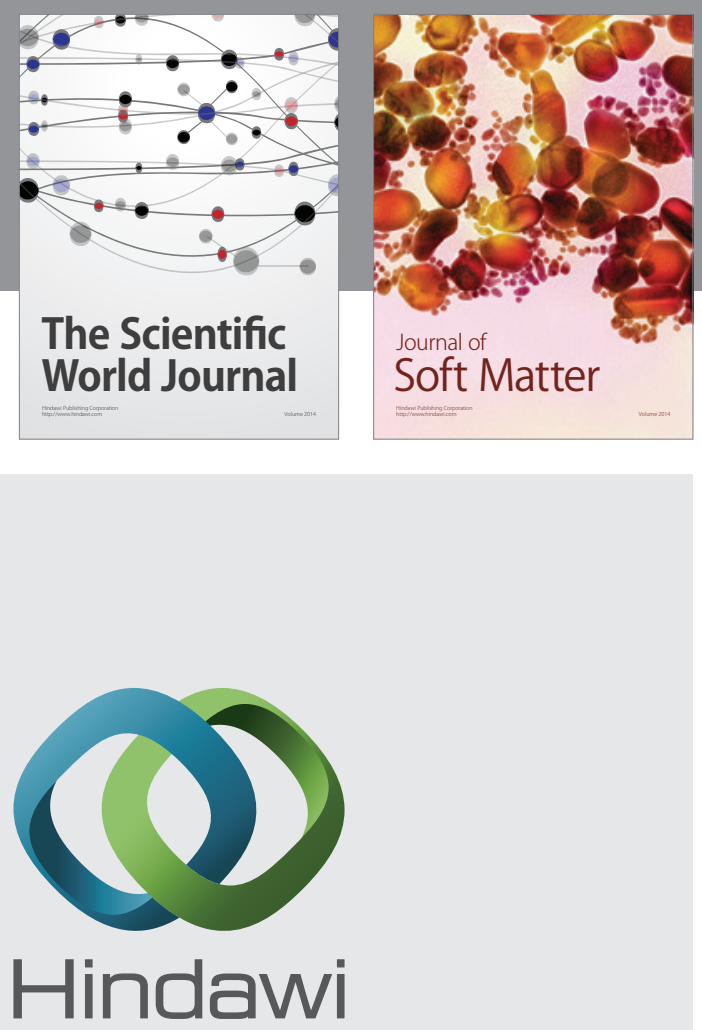

Submit your manuscripts at

http://www.hindawi.com

nternational Journal of

Statistical Mechanics
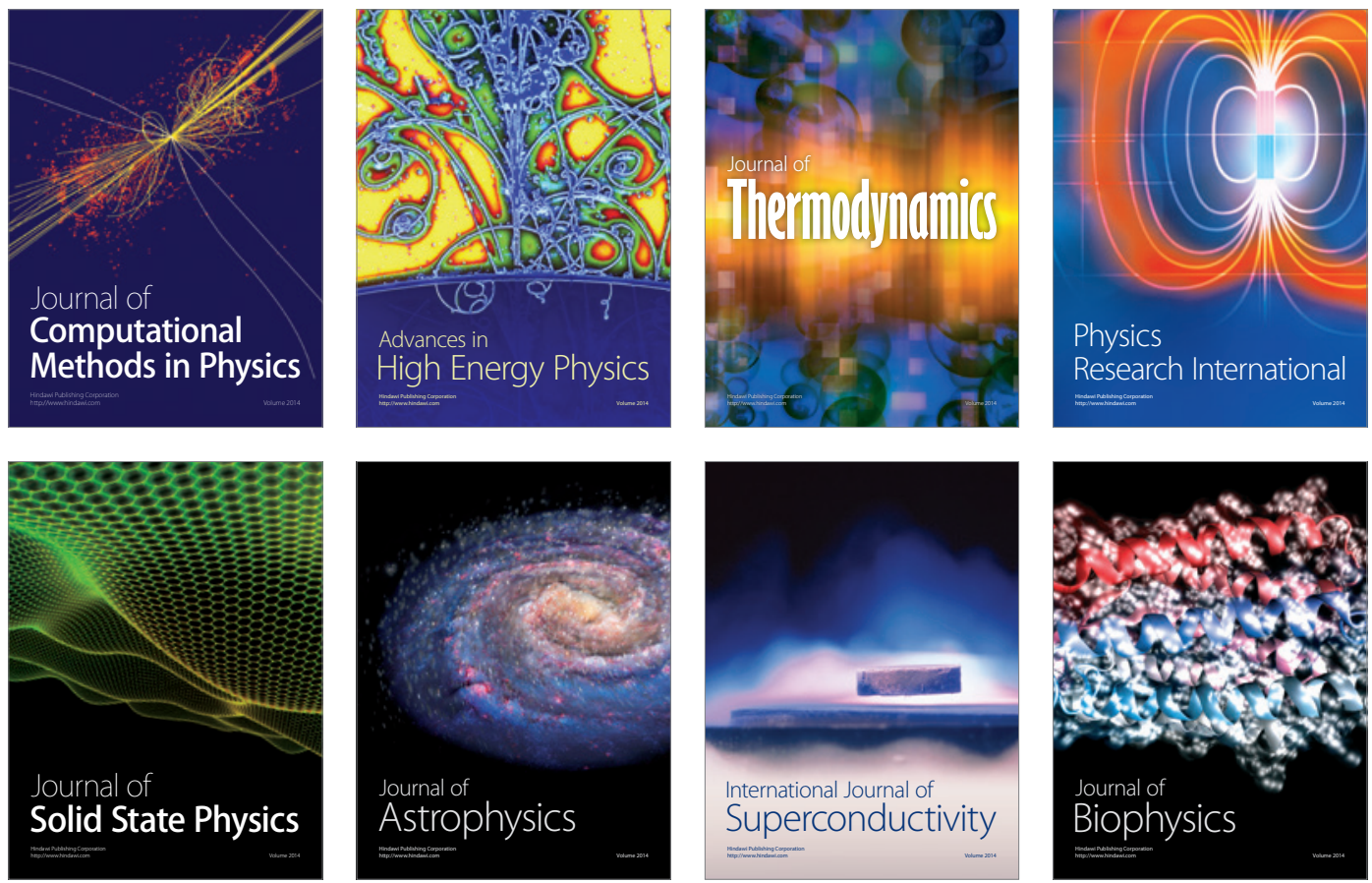
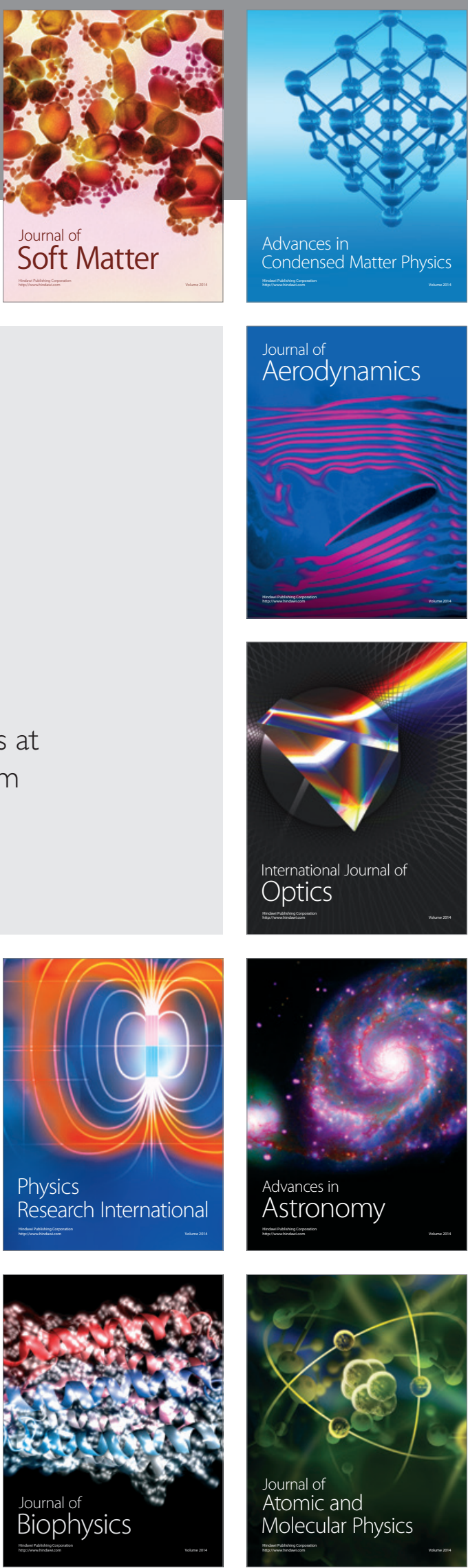